\title{
Hysteresis and hierarchies: dynamics of disorder-driven first-order phase transformations
}

\author{
James P. Sethna, Karin Dahmen, Sivan Kartha, \\ James A. Krumhans ${ }^{\dagger}$,Bruce W. Roberts, and Joel D. Shore* \\ Laboratory of Atomic and Solid State Physics, \\ Cornell University, Ithaca, New York 14853-2501
}

\begin{abstract}
We use the zero-temperature random-field Ising model to study hysteretic behavior at first-order phase transitions. Sweeping the external field through zero, the model exhibits hysteresis, the return-point memory effect, and avalanche fluctuations. There is a critical value of disorder at which a jump in the magnetization (corresponding to an infinite avalanche) first occurs. We study the universal behavior at this critical point using mean-field theory, and also present preliminary results of numerical simulations in three dimensions.

PACS numbers: 75.60.E, 64.60.Ht, 64.60.My, 81.30.Kf, 05.50.+q
\end{abstract}

$\dagger$ Present Address: 515 Station Rd., Amherst, Massachusetts 01002

* Present Address: Department of Physics, Simon Fraser University, Burnaby, British Columbia, Canada V5A 1S6 
First-order phase transitions have always been the weak sibling of critical phenomena in statistical mechanics. At some critical temperature $T_{c}$, the idealized thermodynamic equilibrium properties of a homogeneous material abruptly shift from (say) liquid to gas, with precursor fluctuations almost entirely absent. The classic model for a first order transition $^{1}$ is the Ising model in an external field $H$ at $T<T_{c}$ : as $H$ passes through zero, the equilibrium magnetization reverses abruptly.

There is an amazing contrast, though, with real first-order transitions as studied by materials scientists and metallurgists (figure 1). A solid material which transforms from one crystalline or magnetic form to another under the influence of temperature, external stress, or applied field often has no sharp transition at all. Hysteresis becomes the key phenomenon. Also, there are the experimental Barkhausen ${ }^{2}$ and return-point memory ${ }^{3}$ effects, which are explained here in terms of avalanches and a hierarchy of metastable states.

The ingredient we will add to the idealized physics picture is disorder. Adding a random field $f_{i}$ at each site of the Ising model

$$
\mathcal{H}=-\sum_{i j} J_{i j} s_{i} s_{j}-\sum_{i} f_{i} s_{i}+H s_{i}
$$

allows us to study the effect of disorder in a first order phase transition. We study this random-field Ising model at zero temperature. As the external field $H$ is changed, each spin will flip when the direction of its total local field

$$
F_{i} \equiv \sum_{j} J_{i j} s_{j}+f_{i}+H
$$

changes. The system transforms from negative to positive magnetization as the field is swept upwards. Related approaches ${ }^{4,5}$ have been useful for studying the Barkhausen effect, 
but they have not discussed the return-point memory and did not attempt to vary the disorder and field to reach a critical point.

The dynamics of the approach to thermal equilibrium in this model has been well studied $^{6}$. However, here we study the athermal dynamical response to an external field, where the transition comprises a series of cluster flips. This model is applicable to many experimental systems where the elementary domains have barriers to flipping large enough that thermal activation can be ignored. For example, martensitic transitions come in two varieties, called isothermal and athermal: the athermal martensites show no transformation if the temperature and external strain are held constant, but transform with a crackling noise as these parameters are changed. Similarly, most useful magnetic memory devices by design do not come to thermal equilibrium!

Simulating the system described in equation (1) at zero temperature yields behavior pleasantly familiar to the experimentalist. Figure 1 shows the hysteresis loop for a threedimensional $30^{3}$ system with nearest-neighbor bond strength $J=1$ and random fields $f_{i}$ with a Gaussian probability distribution with a half-width $R$. The outer loop shows the external field $H$ being swept from a large negative value (saturating all spins to -1 ) to a large positive value and back.

The inner loops in figure 1 illustrate the return-point memory effect, seen in some but by no means all first order transitions ${ }^{3}$. (Some first-order transitions exhibit a drift in their hysteretic behavior. ${ }^{5}$ ) If the field $H$ is made to backtrack from $H_{B}$ to $H_{C}$, when it returns to $H_{B}$ the system returns precisely to the same state from which it left the outer loop, it remembers the former state. The slope of the $M(H)$ curve has a nonanalyticity as it rejoins the outer loop: when the field $H(t)$ passes through the previous local maximum $H_{B}$, new spins begin to flip, leading to the apparent slope discontinuity at point $\mathbf{B}$. This same memory effect extends to subcycles within cycles (and so on); the state of the system can remember an entire hierarchy of turning points in its past external field $H(t)$. 
This memory effect is vividly illustrated by experiments measuring the avalanche fluctuations during the transformation. The nucleation of the individual domains in martensites can produce observable pulses in acoustic emission and latent heat. ${ }^{7}$ Under a cycle like that between $H_{B}$ and $H_{C}$ in figure 1, the acoustic emission is resolved into hundreds of individual pulses each lasting some $\mu$ sec, of varying height, forming a "fingerprint" of the cycle. On repeating the cycle, the avalanche structure is precisely reproduced!

We can explain the return-point memory effect theoretically using Alan Middleton's "No Passing" rule, introduced in the study of sliding charge-density waves. ${ }^{8}$ Consider the natural partial ordering of the states: a state $\mathbf{s}=\left\{s_{1}, \ldots, s_{N}\right\} \geq \mathbf{r}=\left\{r_{1}, \ldots, r_{N}\right\}$ if $s_{i} \geq r_{i}$ for each site $i$ in the system. This ordering is not very discriminating: most arbitrary pairs of states will not have any definite relationship. For example, in figure 1, the states a and $\mathbf{b}$ are unrelated: despite the fact that the net magnetization of $\mathbf{a}$ is smaller than that of $\mathbf{b}$, there is likely at least one spin that has flipped on the way from $\mathbf{a}$ to $\mathbf{B}$ which has not flipped back down on the way down to $\mathbf{b}$. This partial ordering becomes important because it is preserved by the dynamics:

No Passing: (Middleton) Let a system $\mathbf{s}(t)$ be evolved under the field $H_{s}(t)$, and similarly $\mathbf{r}(t)$ evolved under $H_{r}(t)$. Suppose the initial configurations satisfy $\mathbf{s}(0) \geq \mathbf{r}(0)$ and the fields $H_{s}(t) \geq H_{r}(t)$. Then it will remain true that $\mathbf{s}(t) \geq \mathbf{r}(t)$ at all times $t$.

Proof: Suppose the contrary. Then there must be some first time that a spin $j$ in $\mathbf{r}$ is going to "pass" the corresponding spin in $\mathbf{s}$, i.e. $r_{j}(t)>s_{j}(t)$. At that time, the local field $F_{j}^{r}$ must be larger than $F_{j}^{s}$. But this cannot be, because the neighbors of $r_{j}$ in state $\mathbf{r}$ at that time are no less negative than those of $s_{j}$ and the external field $H_{r} \leq H_{s}$.

Having established the No Passing property of the system, we make the additional assumption that the system dynamics is adiabatic: the field changes slowly enough that if we start in some state $\mathbf{A}$ any monotonic path from field $H_{A}$ to $H_{B}$ will cause the state 
to evolve in the same way, and into the same final state B. For this model and for other systems with partial ordering, No Passing, and adiabaticity, we can now prove:

Return-Point Memory: Suppose a system s(0) is evolved under field $H(t)$, where $H(0) \leq H(t) \leq H(T)$ for $0<t<T$, with $H(t)$ not necessarily monotonic. Then the final state of the system depends only on $H(T)$, and is independent of the time $T$ or the history $H(t)$. In particular, a system coming back to a previous extremal field will return to exactly the same state, provided that the field remains within these bounds.

Proof: Consider the fields $H_{\min }(t)=\min _{t^{\prime} \geq t} H\left(t^{\prime}\right)$ and $H_{\max }(t)=\max _{t^{\prime} \leq t} H\left(t^{\prime}\right)$. These fields irregularly but monotonically rise from $H(0)$ to $H(T)$. If $\mathbf{s}_{\min }$ evolves under $H_{\min }$ and similarly $\mathbf{s}_{\max }$ evolves under $H_{\max }$, then since $H_{\min }(t) \leq H(t) \leq H_{\max }(t)$ the No Passing rule implies $\mathbf{s}_{\min }(t) \leq \mathbf{s}(t) \leq \mathbf{s}_{\max }(t)$. But by the adiabaticity assumption, all monotonic paths lead to the same final state $\mathbf{s}_{\min }(T)=\mathbf{s}_{\max }(T)$, so $\mathbf{s}(T)$ is independent of path.

Historically, ours is not the first model to exhibit hysteresis and return-point memory. These phenomena are often described by Preisach models ${ }^{9}$, where the system is decomposed into independent elementary hysteresis domains, each with an upper and lower critical field for flipping. Preisach models have the three properties needed for return-point memory, and indeed can be thought of as zero-dimensional variants of our model. They are successful because they demonstrate return-point memory, and because the distribution of domains can be varied in order to fit experimental hysteresis loops. The hypothesis of independent domains, of course, is an idealization, since there is generally some non-trivial coupling between components of the system. As an illustration of the collective behavior missing in the Preisach model, we unearth critical fluctuations and universality buried in the dynamics of the interacting system.

Physically, if the nearest-neighbor coupling is substantial compared to the randomness, i.e. bond strength $J \gg R$, then the system will look like what one expects of a clean first 
order transition: the first sufficiently large region to nucleate will push most of the rest of the sample over the brink. In this small disorder regime, there will be an "infinite avalanche": some spin flips, triggering its neighbors to flip (and so on) until a finite fraction of the sample is transformed, causing a jump in the magnetization density. This infinite avalanche will perhaps be surrounded by precursors and aftershocks. On the other hand, in the large disorder regime, where $J \ll R$ the spins will essentially flip on their own, each spin flipping as the external field crosses its local random field. Those avalanches which occur will be small: there will be no diverging correlation lengths.

Consider the "phase diagram" of our model as we vary disorder $R$ and field $H$ (at $T=0$ ), starting from the state with all spins down (figure 2). There must be a strength of disorder $R_{c}$ above which an infinite avalanche never happens. In contrast, for weaker disorder, there will be a field $H_{c}^{u}(R)$ at which (on raising the field from the down state) an infinite avalanche occurs. Both experimentally ${ }^{10}$ and in our initial simulations in three dimensions, the transition at $H_{c}^{u}(R)$ seems to be abrupt for $R<R_{c}$. (We'd call it firstorder, except the avalanche is supposed to be mediating a first-order transition — the language is failing us.) On the other hand, as one approaches the critical field $H_{c}^{u}\left(R_{c}\right)$ at the critical disorder $R_{c}$, the transition appears to be continuous: the magnetization $M(H)$ has a power-law singularity, and there are avalanches of all sizes. As one approaches this "endpoint" at $\left(R_{c}, H_{c}^{u}\left(R_{c}\right)\right)$ in the $(R, H)$ plane, we find diverging correlation lengths and presumably universal critical behavior.

We can solve for these critical properties exactly within mean-field theory. Suppose every spin in equation (1) is coupled to all $N$ other spins with coupling $J / N$. The effective field acting on a site is $J M+f_{i}+H$, where $M=\sum_{i} s_{i} / N$ is the average magnetization. Spins with $f_{i}<-J M-H$ will point down, the rest will point up. Thus the magnetization 
$M(H)$ is given implicitly by the expression

$$
M(H)=1-2 \int_{-\infty}^{-J M(H)-H} \rho(f) d f
$$

where $\rho(f)$ is the probability distribution for the random field. This equation has a singlevalued solution unless $R \leq R_{c}$ (which in the case of a Gaussian distribution corresponds to $\rho(0) \geq 1 / 2 J)$, at which point hysteresis and an infinite avalanche begin. Near the endpoint, the jump in the magnetization $\Delta M$ due to the avalanche scales as $r^{\beta}$, where $r \equiv\left(R_{c}-R\right) / R_{c}$ and $\beta=1 / 2$. As one varies both $r$ and the reduced field $h \equiv\left(H-H_{c}^{u}\left(R_{c}\right)\right)$, the magnetization scales as $M(h, r) \sim|r|^{\beta} \mathcal{M}_{ \pm}\left(h /|r|^{\beta \delta}\right)$, where \pm refers to the sign of $r$. In mean-field theory $\delta=3$ and $\mathcal{M}_{ \pm}$is given by the smallest real root $g_{ \pm}(y)$ of the cubic equation $g^{3} \mp \frac{12}{\pi} g-\frac{12 \sqrt{2}}{\pi^{3 / 2} R_{c}} y=0$.

Unfortunately, the mean field theory predicts unphysical behavior in two ways. First, there is no hysteresis apart from the infinite avalanche. This is an artifact of the Ising mean-field theory, and is not observed in finite dimensions nor in an otherwise equivalent soft-spin mean-field model. Second, the approach to the infinite avalanche upon varying $H$ for $R<R_{c}$ is continuous in mean-field theory. However, in three dimensions we numerically observe a discontinuous transition, although fluctuation effects do seem to be large.

More interesting is the avalanche size distribution near the critical point, (inset, figure 2). We can solve exactly for the probability $D(s, t)$ of having an avalanche of size $s$, where $t \equiv 2 J \rho(-J M-H)-1$ measures the distance to the infinite avalanche at $\rho=1 / 2 J$. To have an avalanche of size $s$ triggered by a spin with random field $f$, you must have precisely $s-1$ spins with random fields in the range $\{f, f+2 J s / N\}$. The probability for this to happen is given by the Poisson distribution. In addition, they must be arranged so that the first spin triggers the rest. This occurs with probability precisely $1 / s$, which we can see by putting periodic boundary conditions on the interval $\{f, f+2 J s / N\}$ and 
noting that there would be exactly one spin of the $s$ which will trigger the rest as a single avalanche. This leads to the avalanche size distribution

$$
D(s, t)=\frac{s^{s-2}}{(s-1) !}(t+1)^{s-1} e^{-s(t+1)} .
$$

To put this in a scaling form, we must first express $t$ as a function of $r$ and $h$ :

$t \sim r\left[1 \mp \frac{\pi}{4} g_{ \pm}\left(h /|r|^{\frac{3}{2}}\right)^{2}\right]$. Using some simple expansions and Stirling's formula, we can then write $D$ in the scaling form

$$
D(s, r, h) \sim s^{-\tau} \mathcal{D}_{ \pm}\left(s /|r|^{-1 / \sigma}, h /|r|^{\beta \delta}\right)
$$

where our mean-field calculation gives $\tau=3 / 2, \sigma=1 / 2$, and the universal scaling function

$$
\mathcal{D}_{ \pm}(x, y)=\frac{1}{\sqrt{2 \pi}} e^{-x\left[1 \mp \frac{\pi}{4} g_{ \pm}(y)^{2}\right]^{2} / 2} .
$$

As usual, we expect the critical exponents $\beta, \tau, \delta$, and $\sigma$ and the scaling functions $\mathcal{M}$ and $\mathcal{D}$ to be independent of many details of the system (and thus the same for theoretical models and real materials), but to depend on dimension, the range of the interaction, and on the symmetries of the order parameter. We intend to extract critical exponents both numerically and via an $\epsilon$-expansion (the upper critical dimension for the closely related charge-density-wave depinning problem is four ${ }^{11}$ ), study finite temperatures, and introduce frustration representing the dipole fields in magnets and the elastic strain in martensites.

Does the critical behavior predicted here exist in the real world? An FeNi alloy showed a crossover from athermal to burst (infinite avalanche) behavior as the grain size was varied. ${ }^{10}$ Grain boundaries are not random fields, but we expect critical fluctuations and scaling where the bursting first occurs. The distribution of avalanches in magnetic systems has been studied, and some preliminary fits to power laws have been made. ${ }^{2}$ Avalanches and hierarchies have been implicit in the literature since the 1920's; power laws and critical scaling are the tools needed for understanding the collective behavior being studied now. 


\section{Acknowledgments}

We acknowledge the support of DOE Grant \#DE-FG02-88-ER45364. JPS would like to thank the Technical University of Denmark and NORDITA for support and hospitality. BWR acknowledges support from the Hertz Foundation. SK acknowledges support from

the Department of Education. We would like to thank J. Ortín, A. Planes, Llouis Mañosa, Teresa Castán, and Oliver Penrose for helpful conversations. 


\section{Figure Captions}

Figure 1: Hysteresis Loop Showing Return-Point Memory. Shown is the magnetization as a function of external field for a $30^{3}$ system with disorder $R=3.5 J$. Note that the system returns to the original curve at exactly the same state B that it left, that the returning curve has an apparent slope discontinuity at $\mathrm{B}$, and that both effects also happen for the internal subloop. Thus a state can have a whole hierarchy of parent states (mothers at increasing fields and fathers at decreasing fields), which are seen as kinks in the corresponding branch of the $H(M)$ curve.

Figure 2: Varying the Disorder. Three $H(M)$ curves for different levels of disorder, for a $60^{3}$ system. Our current estimate of the critical disorder is $R_{c}=2.21 J$ (we set $J=1$ ). At $R=2<R_{c}$, there is an infinite avalanche which seems to appear abruptly. For $R=$ $2.6>R_{c}$ the dynamics is macroscopically smooth, although of course microscopically it is a sequence of sizable avalanches. At $R=2.3$, near the critical level of disorder, extremely large events become common. Inset: Log-Log Plot of the avalanche-size distribution $D(s)$ vs. avalanche size $s$ for the $60^{3}$ system at $R=2.3$ for $1.3<H<1.4$, averaged over 20 systems. Here $D(s) \sim s^{-1.7}$, compared to the mean-field exponent $\tau$ of $3 / 2$. We expected to see a cutoff in the avalanche sizes: presumably we will see it farther from the endpoint or for larger system sizes. 


\section{REFERENCES}

1. V. Privman and M. E. Fisher, J. Stat. Phys., 33, 385 (1983), M. E. Fisher and V. Privman, Phys. Rev. B 32, 447 (1985), and references therein.

2. J. C. McClure, Jr. and K. Schröder, CRC Crit. Rev. Solid State Sci. 6, 45 (1976); P. J. Cote and L. V. Meisel, Phys. Rev. Lett. 67, 1334 (1991).

3. Return point memory has been seen in ferromagnetism: D. C. Jiles, D. L. Atherton, J. Appl. Phys. 55, 2115 (1984); Barker et al., Proc. R. Soc. London A 386, 251261 (1983). Martensitic transformations (thermally and stress-induced): J. Ortín $J$. Appl. Phys. 71, 1454 (1992); J.de Phys. IV, colloq. C4, 1, C-65, (1991) and references therein. Adsorption of gases: J. Katz, J. Phys. (Colloid) Chem. 53, 1166 (1949); Emmett and Cines, J. Phys. (Colloid) Chem., 51, 1248 (1947). Charge-density waves: Z. Z. Wang and N. P. Ong, Phys. Rev. B 34, 5967 (1986).

4. G. Bertotti and M. Pasquale, J. Appl. Phys. 67, 5255 (1990); J. Appl. Phys. 69, 5066 (1991). J. V. Andersen and O. G. Mouritsen, Phys. Rev. A 45, R5331 (1992).

5. L. P. Lévy, "Reptation and hysteresis in disordered magnets," to be published.

6. J. Villain, Phys. Rev. Lett. 29, 6389 (1984), G. Grinstein and J. F. Fernandez, Phys. Rev. B 29, 6389 (1984), A. J. Bray and M. A. Moore, J. Phys. C 18, L927 (1985), D. S. Fisher, Phys. Rev. Lett. 56, 416 (1986).

7. A. Amengual, Ll. Mañosa, F. Marco, C. Picornell, C. Segui, V. Torra, Thermochimica Acta, 116, 195 (1987); A. Planes, J.L. Macqueron, M. Morin, G. Guenin, L. Delaey, J.de Phys., colloq. C4, 12, C4-615, (1982).

8. A. A. Middleton, Phys. Rev. Lett. 68, 670 (1992); D. S. Fisher and A. A. Middleton, preprint. 
9. F. Preisach, Z. Phys. 94,277 (1935); M. Krasnoselskii and A. Pokrovskii, Systems with Hysteresis, (Nauka, Moscow,1983), I. D. Mayergoyz, J. Appl. Phys. 57, 3803 (1985); Mathematical Models of Hysteresis, (Springer-Verlag,1991); P.C. Clapp, Materials Science and Engineering A127, 189-95 (1990).

10. V. Raghavan in Martensite, G. B. Olson and W. S. Owen, eds., (ASM International), 1992, p. 197.

11. O.Narayan and D.S.Fisher, Phys. Rev. Lett. 68, 3615 (1992) and preprint (unpublished). 